\title{
Lipidomics Analysis of Postmortem Interval: Preliminary Evaluation of Human Skeletal Muscle
}

\author{
Paul L Wood ${ }^{1 *}$ and Natalie R Shirley ${ }^{2}$
}

${ }^{1}$ Metabolomics Unit, Department of Physiology and Pharmacology, Lincoln Memorial University, USA

${ }^{2}$ Department of Anatomy, DeBusk College of Osteopathic Medicine, Lincoln Memorial University, USA

\begin{abstract}
Background: Biochemical determinations of human postmortem interval have focused on acute biomarkers of alterations in metabolites and in microbial generation of amino acids, organic acids, and volatile amines and acids. The need for a reliable biomarker of tissue deterioration on a longer time scale is clear. To determine if structural glycerophospholipids might be of value in this regard, we undertook a lipidomics evaluation of human skeletal muscle at several intervals postmortem.

Methods: Human anterior quadriceps muscle was excised at 1, 9, and 24 days postmortem. Tissue was extracted with tert butyl methylether and methanol and the extracts submitted to shotgun lipidomics analysis.

Results: Sterol sulfates, very-long-chain fatty acids, choline plasmalogens, ethanolamine plasmalogens, and phosphatidylglycerols all were found to decline over the 24 day postmortem period. Free fatty acids were found to increase over the same time period.

Conclusion: Tissue degradation over time postmortem appears to be reliably monitored by the decline in complex structural glycerophospholipids. The contribution of microbes to this degradation remains to be defined. These preliminary data support further evaluations of this lipidomics approach.
\end{abstract}

Keywords: Shotgun lipidomics; Postmortem interval; Plasmalogens; Very-long-chain fatty acids; Phosphatidylglycerol; Biomarkers

\section{Introduction}

The postmortem changes that occur in a corpse are incredibly complex, involving metabolic changes in the corpse, in the endogenous microbiome, and in exogenous invasive microbes. While there are a number of biochemical parameters that have been used to estimate short postmortem intervals, limited efforts have been applied to investigating longer postmortem intervals. Such biochemical parameters would be valuable for practicing forensic anthropologists, medical examiners, DMORT (Disaster Mortuary Operations Response Teams), law enforcement agencies, and human rights investigators. Previous biochemical analyses have focused on tissue amino and organic acid levels [1-4], aqueous humor potassium $[4,5]$, and 3-methoxytyramine in the putamen [5,6], a metabolite of dopamine that is released postmortem [7,8], and proteins [9]. There also are publications of the analysis of volatile organics in soil around a corpse [10-13]. However, such biochemical parameters are extremely variable and difficult to reliably quantitate without rapid transfer of samples in sealed containers to the appropriate analytical facilities. Postmortem biochemical studies are complicated by the multiple sources of metabolites. These include metabolites from the corpse, the GI flora of the corpse, and invasive microbes after death. These diverse metabolic sources make the interpretation of amino and organic acid analyses difficult. We hypothesized that, in marked contrast to these complicated interpretations, lipidomic biomarkers of membrane degradation might have the potential to more accurately predict the rate of postmortem tissue decay. The investigation of human structural lipids that degrade postmortem is a research area that has not been investigated to-date and represents new data for building a post-mortem biochemical database. We therefore undertook a pilot study utilizing high resolution mass spectrometry platforms to examine potential biomarkers of time-dependent postmortem tissue degradation. New lipidomics analytical platforms allow for detailed analyses of these processes. Utilization of lipidomics in postmortem studies offer the potential to generate data to build the first comprehensive database of changes in the lipidome in diverse biochemical pathways and in structural biomolecules that have never been assayed before in postmortem studies. Structural glycerophospholipids (GPL) are one of the most abundant lipid families that are critical for membrane and organelle integrity. The breakdown of these structural lipids is a slow process and was anticipated to yield new biomarkers of postmortem tissue degradation.

\section{Materials and Methods}

\section{Postmortem samples}

Samples (10-14 mg wwt) of the anterior quadriceps were excised from a corpse stored at $34^{\circ} \mathrm{F}$ at the Forensic Anthropology Center at the University of TN, Knoxville. Samples were taken at 1, 9, and 24 days postmortem and frozen for transfer to the analytical facilities at LMU-DCOM. Muscle was processed via sonication utilizing tert- butyl methylether and methanol for extraction of lipids $[14,15]$. The extraction solution contained $\left[{ }^{2} \mathrm{H}_{8}\right]$ arachidonic acid, $\left[{ }^{2} \mathrm{H}_{4}\right]$ hexacosanoic acid, $\left[{ }^{2} \mathrm{H}_{31}\right]$ PtdEtn 34:1, $\left[{ }^{2} \mathrm{H}_{31}\right] \mathrm{PtdCh} 34: 1$, and $\left[{ }^{2} \mathrm{H}_{31}\right] \mathrm{PG} 34: 1$ as internal standards (Avanti Polar Lipids). Extracts were dried by centrifugal vacuum

*Corresponding author: Paul L Wood, Metabolomics Unit, Department of Physiology and Pharmacology, 6965 Cumberland Gap Pkwy, Harrogate, TN 37752, USA, Tel: 423-869-6666; E-mail: paul.wood@Imunet.edu

Received July 25, 2013; Accepted October 21, 2013; Published October 22 2013

Citation: Wood PL, Shirley NR (2013) Lipidomics Analysis of Postmortem Interval: Preliminary Evaluation of Human Skeletal Muscle. Metabolomics 3: 127. doi:10.4172/2153-0769.1000127

Copyright: (C) 2013 Wood PL, et al. This is an open-access article distributed under the terms of the Creative Commons Attribution License, which permits unrestricted use, distribution, and reproduction in any medium, provided the original author and source are credited. 
evaporation and dissolved in isopropanol:methanol:chloroform 4:2:1 containing $7.5 \mathrm{mM}$ ammonium acetate. Shotgun lipidomics were performed utilizing high-resolution (140,000 at $200 \mathrm{amu}$; sub-ppm mass accuracy) data acquisition on an orbitrap mass spectrometer (Thermo Q Exactive), with successive switching between polarity modes [15]. Washes between samples with hexane/ethyl acetate (3:2) were used to minimize ghost effects. In negative ESI, the anions of ethanolamine plasmalogens, phosphatidylglycerols, and fatty acids were quantitated and lipid identities validated by MS/MS [15]. In positive ESI, the cations of choline plasmalogens were quantitated and lipid identities validated by MS/MS [15].

\section{Data analysis}

Metabolite levels are expressed as nmol or pmol per mg wet weight. In cases of compounds for which we did not have analytical standards, the ratio $(\mathrm{R})$ to an appropriate internal standard was used and corrected for tissue wet weight.

\section{Results}

\section{Sterol sulfates}

Sterol sulfates are metabolic pools that serve as reservoirs of sterols in humans. These were anticipated to decline postmortem, and this was found to be the case with cholesterol sulfate and dehydroepiandrosterone sulfate (DHEA sulfate) (Figure 1). DHEA sulfate is the major circulating steroid hormone in humans.

\section{Short Chain Fatty acids and Very-Long-Chain Fatty Acids (VLCFA)}

Short chain fatty acids are major energy sources for both resident and invading microbes in a corpse. We anticipated that these fatty acids would increase with tissue deterioration, both from the breakdown of tissue lipid stores and microbial metabolism. This was found to be the case with arachidonic acid and the associated fatty acid, sciadonic acid (Figure 2).

VLCFA are the products of complex lipid metabolism in humans and decrease with postmortem interval as demonstrated for hexacosanoic (26:0) and octacosanoic (28:0) acids (Figure 2).

\section{Ethanolamine Plasmalogens (PlsEtn) \& Choline Plasmalogens (PlsCh)}

PlsEtn are major components of cell membranes and the membranes of cellular organelles. These complex lipids were found to decrease (Figure 3 ) with postmortem tissue degradation as depicted for PlsEtn(18:0/18:1) and PlsEtn(18:0/22:6).

PlsCh also are major components of cell membranes and the membranes of cellular organelles. These complex lipids decrease with postmortem tissue degradation (Figure 3).

\section{Phosphatidylglycerols}

We also monitored time-dependent decreases in muscle levels phosphatidylglycerols Figure: 4, which are structural GPL and precursors to mitochondrial cardiolipins.

\section{Discussion}

It simply cannot be overstated or overemphasized that the landmark Daubert v. Merrell-Dow Pharmaceuticals, Inc. decision forever changed the face of the forensic sciences in terms of research, practice, and policy. This decision mandates that scientific knowledge is derived from empirical testing using scientific methods that are based on reliable principles and methods [16,17]. Scientific researchers must ensure that methods are sufficiently relevant, robust, and reliable to satisfy admissibility standards. Postmortem interval (PMI) estimates made by examining gross morphological changes in tissues (color changes, skin slippage, bloating, marbling, etc.) are subjective, lack quantification and error rates, and are subject to significant error in the event that the decompositional environment is unfamiliar or unknown. In this study we utilized high resolution mass spectrometry to perform shotgun lipidomics analyses of muscle samples. These are established methods for the lipidomics analysis of complex biological samples [15]

We obtained exciting preliminary data of changes in a number of lipid metabolites that have not been reported previously for postmortem studies. The declines that we monitored in structural glycerophospholipids are consistent with our hypothesis that these lipids would undergo slower degradation profiles and may ultimately provide a more accurate index of postmortem interval.

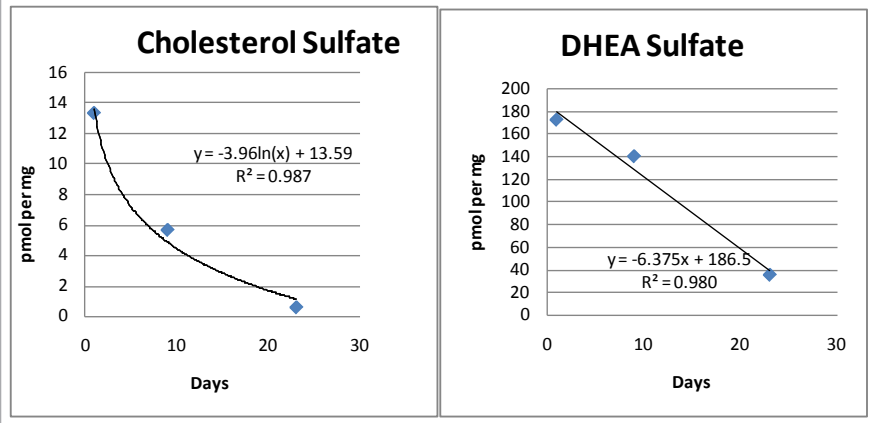

Figure 1: Postmortem decline in muscle levels of cholesterol- and dehydroepiandrosterone (DHEA)-sulfates.


Figure 2: Postmortem accumulation of polyunsaturated fatty acids (arachidonic and sciadonic) and decline in very-long-chain fatty acids (VLCFA). 
Citation: Wood PL, Shirley NR (2013) Lipidomics Analysis of Postmortem Interval: Preliminary Evaluation of Human Skeletal Muscle. Metabolomics
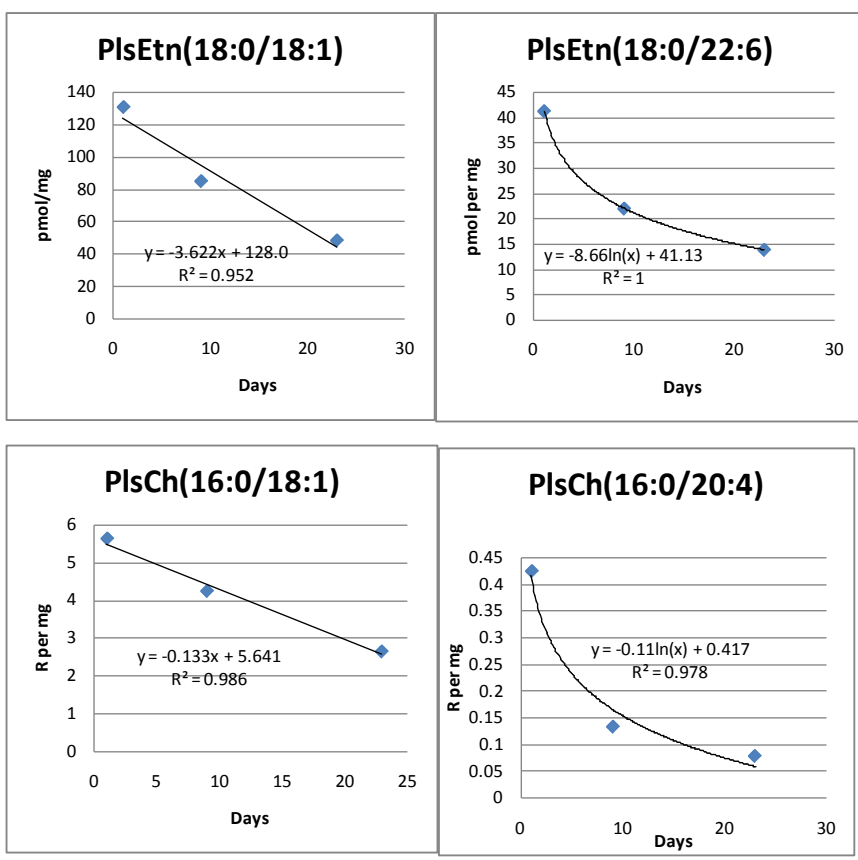

Figure 3: Postmortem decline in muscle plasmalogen levels. PlsCh, choline plasmalogen; PIsEtn, ethanolamine plasmalogen; 16:0, palmitate; 18:0, stearate; $18: 1$, oleate; $20: 4$, arichidonate; $22: 6$, docosahexaenoate.



Figure 4: Postmortem decline in phosphatidylglycerol (PG) levels.16:0, palmitate; 18:0, stearate

In summary, the use of a shotgun lipidomics assay platform generated the quantitative data required to drive a lipidomics analysis of skeletal muscle tissue during postmortem tissue degradation and ultimately to provide more accurate and robust biomarkers of postmortem interval for forensic studies. This preliminary analysis has demonstrated that lipodomic analyses of postmortem tissues are suitable to provide quantitative, objective PMI estimates with associated error terms. With more sampling points we will be able to generate superior regression curves (linear and/or nonlinear, as deemed appropriate) for the time courses in the degradation of individual glycerophospholipids. In addition, sampling of multiple tissues will provide a better definition of optimal biomarkers and optimal tissues for sampling. This approach has the potential to establish reliable biochemical criteria for postmortem interval over a long postmortem period and in varying environmental conditions.

\section{Acknowledgements}

We are grateful to the individual who donated their remains to the Forensic Anthropology Center at the University of TN, Knoxville for research purposes. We also wish to thank the center for collaborating on this pilot study.

\section{References}

1. Vass AA, Barshick SA, Sega G, Caton J, Skeen JT, et al. (2002) Decomposition chemistry of human remains: a new methodology for determining the postmortem interval. J Forensic Sci 47: 542-553.

2. Bonte W, Bleifuss J (1977) Postmortem dating of putrefied material through ptomaine estimation. J Forensic Sci 22: 558-572.

3. Ith M, Scheurer E, Kreis R, Thali M, Dirnhofer R, et al. (2011) Estimation of the postmortem interval by means of ${ }^{1} \mathrm{H}$ MRS of decomposing brain tissue: influence of ambient temperature. NMR Biomed 24: 791-798.

4. Madea B, Musshoff F (2007) Postmortem biochemistry. Forensic Sci Int 165 165-171.

5. Sparks DL, Oeltgen PR, Kryscio RJ, Hunsaker JC 3rd (1989) Comparison of chemical methods for determining postmortem interval. J Forensic Sci 34: 197206.

6. Sparks DL, Slevin JT, Hunsaker JC 3rd (1986) 3-Methoxytyramine in the putamen as a gauge of the postmortem interval. J Forensic Sci 31: 962-971.

7. Wood PL, Kim HS, Marien MR (1987) Intracerebral dialysis: direct evidence for the utility of 3-MT measurements as an index of dopamine release. Life Sci 41: 1-5.

8. Wood PL, Altar CA (1988) Dopamine release in vivo from nigrostriatal mesolimbic, and mesocortical neurons: utility of 3-methoxytyramine measurements. Pharmacol Rev 40: 163-187.

9. Bonte W, Bleifuss J, Volck J (1976) Experimental investigations in post-mortem protein degradation. Forensic Sci 7: 9-22.

10. Vass AA, Bass WM, Wolt JD, Foss JE, Ammons JT (1992) Time since death determinations of human cadavers using soil solution. J Forensic Sci 37: 12361253.

11. Vass AA, Smith RR, Thompson CV, Burnett MN, Dulgerian N, et al. (2008) Odor analysis of decomposing buried human remains. J Forensic Sci 53: 384391.

12. Vass AA (2012) Odor mortis. Forensic Sci Int 222: 234-241.

13. Cablk ME, Szelagowski EE, Sagebiel JC (2012) Characterization of the volatile organic compounds present in the headspace of decomposing animal remains, and compared with human remains. Forensic Sci Int 220: 118-125.

14. Wood PL, Khan MA, Smith T, Ehrmantraut G, Jin W, et al. (2011) In vitro and in vivo plasmalogen replacement evaluations in rhizomelic chrondrodysplasia punctata and Pelizaeus Merzbacher disease using PPI-1011, an ether lipid plasmalogen precursor. Lipids Health Dis 10: 182

15. Schuhmann K, Almeida R, Baumert M, Herzog R, Bornstein SR, et al. (2012) Shotgun lipidomics on a LTQ Orbitrap mass spectrometer by successive switching between acquisition polarity modes. J Mass Spectrom 47: 96-104.

16. Christensen AM (2004) The impact of Daubert: implications for testimony and research in forensic anthropology (and the use of frontal sinuses in personal identification). J Forensic Sci 4: 427-430.

17. Grivas CR, Komar DA (2008) Kumho, Daubert, and the nature of scientific inquiry: implications for forensic anthropology. J Forensic Sci 53: 771-776. 